Aim: To determine the effectiveness of interdisciplinary prehospital simulation workshops in preparing volunteers for MAT deployment at MGEs.

Methods: A one-day, simulation-based training session within the MAT environment was implemented to introduce volunteers to the management of various scenarios faced at MGEs. All volunteers were provided an orientation to the equipment and setting up MAT deployments at MGEs. Volunteers then participated in interdisciplinary group-based scenarios such as cardiac arrest management, drug intoxication, spinal injuries, agitated patients, and airway management. To determine the effectiveness of this training session, volunteers were invited to participate in a post-training survey, comprising of Likert scores and open-ended responses.

Results: Seventeen volunteers attended the training session with 10 (58.8\%) completing the post-training survey. Volunteers were satisfied with environment familiarization in the MAT (Average 4.47/5.00) and found the simulation-based training helpful (Average 3.67/4.00). The induction overall was well-received (4.60/5.00) with volunteers feeling more confident in being deployed at MGEs (4.20/5.00).

Discussion: The results of the simulation-based training session were positive with volunteers receptive to the need for a training day prior to MAT deployment at MGEs. The simulation session enables volunteers to be comfortable with working in MAT and managing a diverse range of patients at MGEs. This session is likely to improve interdisciplinary communication and teamwork in the MAT. Future research is aimed at following these volunteers after several MAT deployments to improve the training session for future participants.

Prehosp Disaster Med 2019;34(Suppl. 1):s102-s103

doi:10.1017/S1049023X19002127

Assessing the Impact of a New Emergency Triage System on Head Injury Mortality: Tikur Anbessa Specialized Hospital Emergency Department in Addis Ababa, Ethiopia Dr. helena Fantaye ${ }^{1}$, Dr. Amanuel Lomencho ${ }^{2}$, Dr. Pol de vos ${ }^{3}$

1 Ministry Of Health, Ethiopia, Addis Abaaba, Ethiopia

2 American Medical Center, Addis Ababa, Ethiopia

3 Institute of Tropical Medicine, Antwerp, Belgium

Introduction: One of the improvements in Ethiopia's emergency medical system was the introduction of a five-level Emergency Triage System (ETS) in January 2015 that was piloted in selected Addis Ababa hospitals.

Aim: To assess the effect of this intervention on the head injury mortality in Tikur Anbessa Specialized Hospital (TASH) Emergency Department (ED).

Methods: Data were retrospectively collected from all medical records of head injury patients seen in Adult TASH- ED over two 6 months periods, before and after the new Emergency Triage System implementation: 01/04/2014 - 30/09/2014 versus $01 / 04 / 2016$ - 30/09/2016. An inclusion criterion was age above 13 for the records that could be retrieved. Exclusion criterion was "patient declared dead on arrival." Mortality and patterns of head injury were compared pre- and post-intervention. Chi-square was used for the analysis using STATA 14.
Results: A total of 522 Head injury patients were analyzed in the ED in both the pre- 258 and post-264 intervention study periods. Among head injury admission in the ED in both study periods, the highest number of patients were Road Traffic Accident/ RTA/ victims, males and young age $(<30)$. Mortality rate among head injury patients decreased from a pre-intervention 44 $(17.05 \%)$ to post-intervention $27(10.2 \%)(\mathrm{OR}=0.559 .5 \% \mathrm{CI}$ $(0.32,0.95), \mathrm{p}=0.02)$. The median age of death was 45 years in pre- and 40 years in the post-intervention period, with ages ranging from 13 to 85 and 13 to 96 years, respectively. The proportion of deaths from moderate head injury decreased significantly from $14.0 \%$ in pre-intervention to $6.3 \%$ in the postintervention period, respectively $(\mathrm{p}<0.001)$.

Discussion: The Emergency Triage System at TASH-ED has decreased mortality caused by head injury. This could increase life years saved and productivity in a cost-effective and easily achievable way in resource-poor settings.

Prehosp Disaster Med 2019;34(Suppl. 1):s103

doi:10.1017/S1049023X19002139

\section{Assessment of Emergency Medical Rescue Ability of Secondary and Tertiary Hospitals in One City Responding to the Risk of Production and Storage of Hazardous Chemicals}

Dr. Xu Hu

West China Hospital, sichuan university, Chengdu, sichuan, China

Introduction: With the development of the economy and the expansion of the hazardous chemicals industry in one city, it is necessary for the city to establish an evaluation model of emergency medical rescue capability for hazardous chemicals production, storage, and exposure risk.

Aim: Establish an emergency medical rescue capacity evaluation model for secondary and higher hospitals in a city to deal with exposure risks of hazardous chemicals.

Methods:

1. Develop an expert consultation form

2. Develop a survey on the status quo of emergency medical rescue capacity of hospitals in secondary and above hospitals in response to exposure and risk of hazardous chemicals production and storage.

3. Calculate the weights of the first, second, and third-grade indicators, and establish a comprehensive evaluation model for the rescue capacity assessment of Chengdu hospitals.

Results: Five levels of first-level indicators were obtained, namely, the weights of the five indicators of "centralized admission ability," "hospital comprehensive ability," "emergency management ability," "material equipment capability," and "health emergency team" were $0.2884,0.2219,0.1938$, 0.1507 , and 0.1453 , respectively.

Discussion: The establishment of an emergency medical rescue capacity evaluation model for the risk of exposure and storage of hazardous chemicals in secondary and higher hospitals in a city is related to five capabilities, the most important of which is the ability to focus on admission.

Prehosp Disaster Med 2019;34(Suppl. 1):s103

doi:10.1017/S1049023X19002140 